\title{
Restoration of finger blood flow oscillations by means of thermal imaging
}

\author{
by A.A. Sagaidachnyi, D.A. Usanov, A.V. Skripal, A.V. Fomin
}

Dept. of Nano - and Biomedical Technology, Saratov State University, Saratov, Russia, andsag@yandex.ru

Abstract

Interrelation of skin temperature and blood flow oscillations of intact finger phalange has been investigated. Oscillations of a blood flow were measured by photoplethysmography, oscillations of a temperature were registered by thermal imaging camera. The considered method of restoration of blood flow oscillations from temperature oscillations shows a good possibility of reconstruction of time-frequency spectrum of blood flow, especially in neurogenic frequency band $0.02-0.05 \mathrm{~Hz}$. Temperature data are lagging in relation to blood flow values and has a time delay specific for each subject. The method of blood flow oscillations restoration can find practical application in investigations of physical and chemical impacts on the circulatory dynamics and in phase coherence investigations.

\section{Introduction}

Results of investigation of skin temperature and blood flow oscillations contain restricted and frequently contradictory details of determination of temperature oscillations by blood flow oscillations. For example, in [1] it was reported about the similarity of the form of blood flow oscillations and temperature oscillations and presence of 20-30 second delay between the signals. On the other hand, in [2] it was said about absence of time delay between slow oscillations of temperature and level of blood flow. Thus, the question about the interrelation between temperature oscillations and local blood flow remains open. What are the possibilities of blood blow restoration by means of temperature measurements? How can best correlations between blood flow and temperature oscillations be achieved?

The aims of the present study are to investigate interrelation between temperature and blood flow oscillations of finger tips in healthy subjects under normal environmental conditions, to define the correlation of the two signals in time and time-frequency domains and possibilities of restoration of blood flow oscillations through temperature data.

There is a well-known method of photoplethysmography (PPG) which measures infrared radiation absorption of blood by hemoglobin that circulates in surface tissues, in order to estimate blood flow oscillations alongside with usage of laser and ultrasonic Doppler methods. Amplitude of PPG depends on microvessels tonus and heart stroke volume. Our investigations are carried out under conditions of minor variations of a heart stroke volume, therefore, changes of PPG amplitude describes variations of blood flow as a result of microvessels tonus variations. Earlier in [3] high correlation of PPG signal and blood flow oscillations has been established (correlation coefficient $r=0.94$ ), that testifies to high opportunities of photoplethysmography in blood flow investigations. It is natural to expect that blood supply oscillations should be accompanied by corresponding temperature oscillations.

Thus, the main interest lies in the investigation of correlation between finger tips temperature oscillations measured by thermal imaging method, blood flow oscillations estimated by means of photoplethysmography, and restoration of blood flow oscillation from temperature data.

\section{Infrared imaging and PPG measurements}

Simultaneous measurements of finger temperature and blood flow oscillations in 11 healthy volunteers under normal environment conditions during 20 minutes were made. The age of the subjects was from 20 to 35 years. Measurements were performed after adaptation of subjects to lab conditions in the course of 10-15 minutes.

A calibrated IR camera (ThermaCam SC 3000, Flir Systems, Sweden) with $0.02^{\circ} \mathrm{C}$ temperature sensitivity $(8-$ $9 \mu \mathrm{m}$ wavelength, $320 \times 240$ pixels per image) was used for the temperature measurements with the sample rate of $2 \mathrm{~Hz}$.

The photoplethysmography was measured by means of the sensor KL-79102 (Biomedical measurement system $\mathrm{KL}-72001$, Taiwan) with the sample rate of $50 \mathrm{~Hz}$.

The volunteers did not consume any tonic or alcoholic beverages before measurements were taken. There were no smokers in the volunteer group. All measurements were carried out in the sitting position, hands immobilized on the table with the surface made of small heat capacity material (foam plastic). All measurements were taken from the distal phalange of an index finger. An index finger was placed on the top of the PPG sensor, the rectangle region of interest for temperature measurements was located at the lateral side of phalange (see Figure 1). Comparison of the temperature and PPG oscillations has been performed both in the time and the time-frequency domains. 


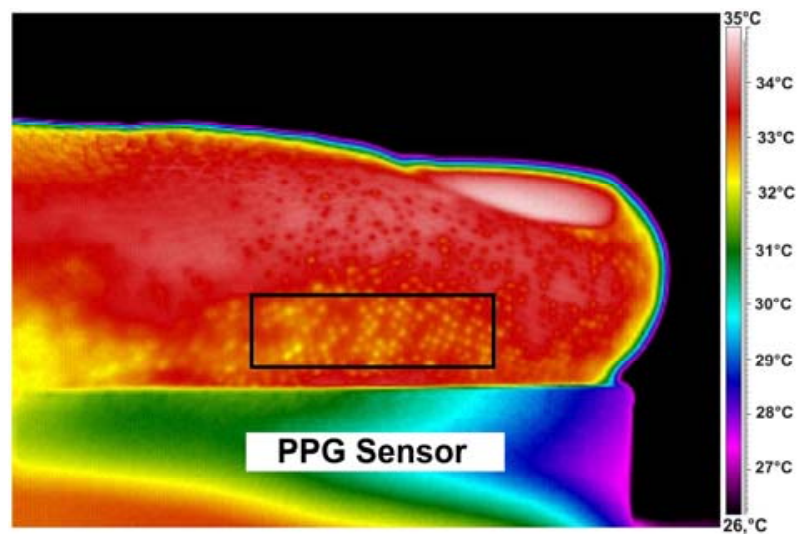

Fig. 1. Regions of interest for temperature and photoplethysmography distal phalange measurements

\section{Time domain analysis} in Figure 2.

The sequence of signal processing of photoplethysmography and temperature data is illustrated by the diagram

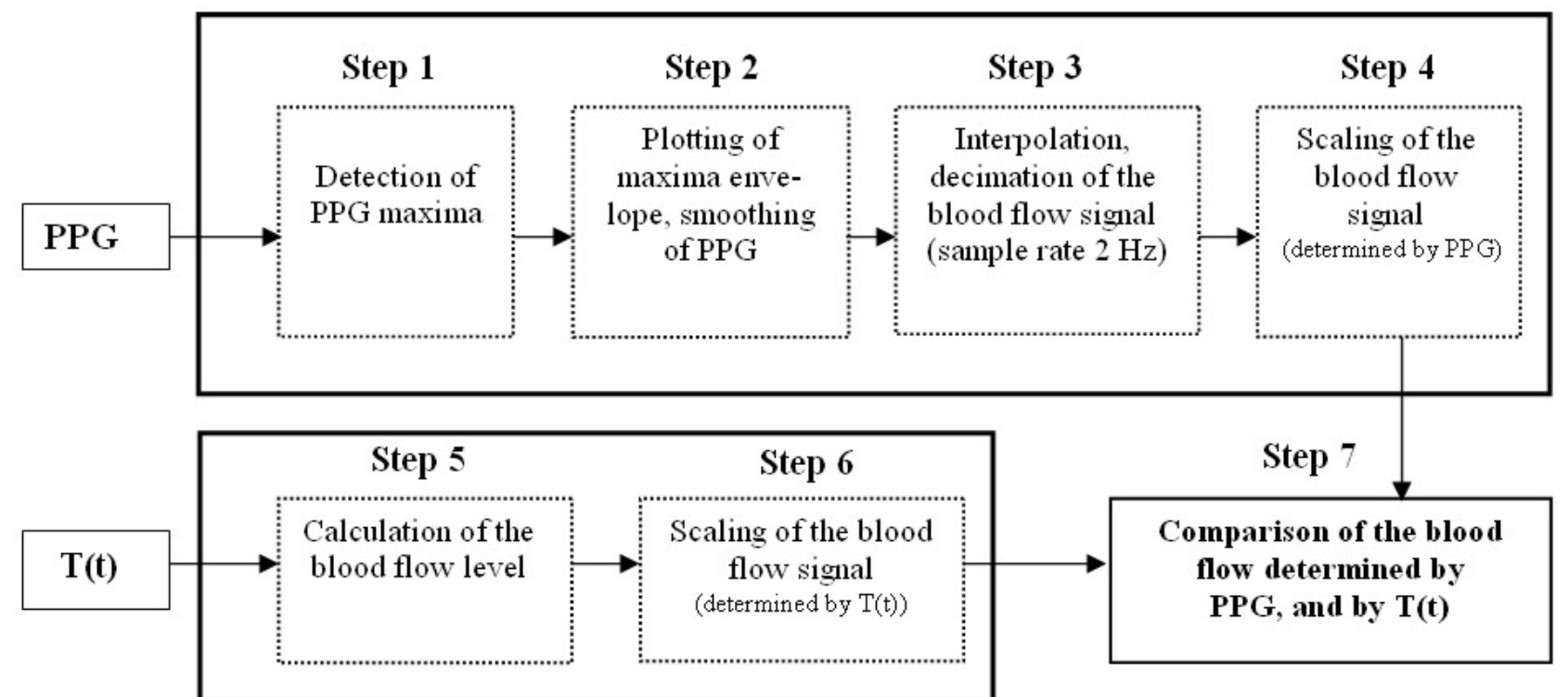

Fig. 2. Processing of the photoplethysmography (PPG) and the temperature $T(t)$ signals

A maxima envelope of PPG was used as a characteristic of a finger blood flow (step 1 in Figure 2). Fourier filtering of low-frequency PPG signal can be an alternative method of slow oscillations detecting [4]. Further, the slowly varying PPG signal smoothed (function ksmooth, MathCAD ) and scaled (step 2 in Figure 2). Linear scaling Eq. (1) of $\mathrm{T}(\mathrm{t})$, and $\mathrm{PPG}$ signals represented values in the range of $[0 ; 1]$.

$$
Y_{\text {norm }}(t)=\frac{Y(t)-Y_{\min }}{Y_{\max }-Y_{\min }},
$$

where $Y_{\min }$ is the minimum value of signal, $Y_{\max }$ is the maximum value of signal, $Y(t)$ is the value of signal in a time $t$.

The equation (2) [5], considers heat loss due to a combination of natural convection and radiation, and heat delivered by blood flow to the tissue, was used for restoration of blood flow oscillations by temperature oscillations.

$$
\rho \cdot C \cdot V \cdot \frac{d T(t)}{d t}=-H_{\text {air }} \cdot S \cdot\left(T(t)-T_{\text {air }}\right)+\rho_{b} \cdot C_{b} \cdot \omega(t) \cdot\left(T_{b}-T(t)\right)+Q_{m},
$$

where $S=\frac{\pi \cdot D^{2}}{2}$ is the surface of the finger, $V=\frac{\pi \cdot D^{3}}{12}$ is the volume of the finger, $t$ is the time, $\rho$ is the tissue density, $\rho_{b}$ is the blood density, $C$ is the specific tissue capacity, $C_{b}$ is the specific blood capacity, $D$ is the diameter of finger, $S$ is the surface of the finger (surface of hemisphere), $T_{b}$ is the blood temperature, $T_{\text {air }}$ is the air temperature, $T(t)$ is the temperature of skin surface, $V$ is the finger volume (hemisphere), $\omega$ is the volume of blood flow, $Q_{m}$ is the quantity 
of metabolic heat, $\mathrm{H}_{\text {air }}$ is the convection coefficient of air. Description of parameters and their used values are given in Table $1[5]$.

If temperature difference between skin and environment increases, the Eq. (2) shows a slowdown of temperature dynamics, and if blood flow and intensity of metabolic heat sources increases, then it shows accelerating.

Table 1. Values of parameters used in Eq. (2)

\begin{tabular}{|c|c|c|}
\hline Symbol & Denomination & Value \\
\hline$\rho$ & tissue density & $1057 \mathrm{~kg} / \mathrm{m}^{3}$ \\
\hline$\rho_{b}$ & blood density & $1069 \mathrm{~kg} / \mathrm{m}^{3}$ \\
\hline $\mathrm{C}$ & specific heat capacity of a tissue & $3780 \mathrm{~J} \cdot \mathrm{K} / \mathrm{kg}$ \\
\hline $\mathrm{C}_{\mathrm{b}}$ & specific heat capacity of a blood & $3650 \mathrm{~J} \cdot \mathrm{K} / \mathrm{kg}$ \\
\hline $\mathrm{D}$ & diameter of a finger & $0.011-0.016 \mathrm{~m}$ \\
\hline $\mathrm{T}_{\mathrm{b}}$ & blood temperature & $37^{\circ} \mathrm{C}$ \\
\hline $\mathrm{T}_{\text {air }}$ & air temperature & $24^{\circ} \mathrm{C}$ \\
\hline $\mathrm{V}$ & finger volume (hemisphere) & $1.072 \cdot 10^{-6} \mathrm{~m}^{3}$ \\
\hline $\mathrm{S}$ & finger surface (hemisphere) & $4.02 \cdot 10^{-4} \mathrm{~m}^{2}$ \\
\hline $\mathrm{H}$ & convection coefficient of air & $6 \mathrm{~W} \cdot \mathrm{K} / \mathrm{m}^{2}$ \\
\hline
\end{tabular}

If there is no intensive muscular work, metabolic component $Q_{m}$ in Eq. (2) can be considered as a negligible small or a constant dc component. In this case the required temperature level is supported through the balance between heat loss (first item in Eq. 2) and heat reception from blood (second item in Eq. 2). In general case, when taking into account the delay between blood flow and temperature signals, it's necessary to input the delay time argument $\Delta \mathrm{t}$ in the Eq. (2). Thus, neglecting the item $Q_{m}$ and applying delay time $\Delta t$, volume blood flow $\omega$ can be expressed from Eq. (2) as:

$$
\omega\left(t_{i}-\Delta t\right)=\frac{\rho \cdot C \cdot V \cdot \frac{d T\left(t_{i}\right)}{d t}+H_{a i r} \cdot S \cdot\left(T\left(t_{i}\right)-T_{a i r}\right)}{\rho_{b} \cdot C_{b} \cdot\left(T_{b}-T\left(t_{i}\right)\right)}
$$

where $t_{i}$ is the time, $i$ is the index of time counts.

To determine the blood flow $\omega$, we substituted temperature values $T\left(t_{i}\right)$ and its derivatives in the right member of Eq. 3 (step 5 in Figure 2). Calculation of time derivatives of temperature was performed by 5 points finite difference method.

Determination of a delay time $\Delta t$ of the temperature variations in relation to volume blood flow was performed by means of cross-correlation function Eq. (4).

$$
F\left(\Delta t_{j}\right)=\sum_{i} \omega_{T}\left(t_{i}+\Delta t_{j}\right) \cdot \omega_{P P G}\left(t_{i}\right)
$$

where $\mathrm{j}$ is the index of time shift.

The value of the delay determined from the maximum of cross-correlation function F. Table 2 shows the results of the delay determination for each subject. In Figure $3 \mathrm{c}$ time shift is added to the temperature signal $\mathrm{T}(\mathrm{t})$, equalling delay time $\Delta \mathrm{t}$ (step 7 in Figure 2 ).

Comparison of the blood flow variations, determined by temperature data - $\omega_{T}(t)$ and by photoplethysmography $\omega_{P P G}(t)$ with the aim of calculation of correlation coefficient in time domain was carried out next (5):

$$
r_{\text {time }}=\frac{\sum_{i}\left(\omega_{T}\left(t_{i}\right)-\bar{\omega}_{T}\right) \cdot\left(\omega_{P P G}\left(t_{i}\right)-\bar{\omega}_{P P G}\right)}{\sqrt{\sum_{i}\left(\omega_{T}\left(t_{i}\right)-\bar{\omega}_{T}\right)^{2} \cdot \sum_{i}\left(\omega_{P P G}\left(t_{i}\right)-\bar{\omega}_{P P G}\right)^{2}}}
$$

where $\bar{\omega}_{T}, \bar{\omega}_{P P G}$ are the average values of volume blood flow, determined by temperature data and by photoplethysmography, respectively (see $r$ values in Table 2).

\section{Time-frequency domain analysis}

In order to observe spectrum changes in the course of substitution the temperature signal to the formula (3), the time-frequency wavelet transform (6) was applied to the signals. The transform (6) is obtained by convolving the signal $\mathrm{f}(\mathrm{t})$ with Morlet wavelet $\psi(\mathrm{t})(7)$, which is relatively high localized both in the time and frequency domains [6]. 


$$
W(a, b)=\frac{1}{a} \cdot \int_{-\infty}^{+\infty} f(t) \cdot \psi\left(\frac{t-b}{a}\right) d t
$$

where $a$ is the scale value which has the dimension of the time and inversely proportional to the frequency of the signal, $\mathrm{b}-$ is the time shift (wavelet position), $\mathrm{t}$ is the dummy variable represented the time of a signal.

$$
\psi(t)=\frac{1}{\sqrt[4]{\pi}} \cdot e^{\left(-i \cdot \omega_{0} \cdot t\right)} \cdot e^{\left(-\frac{t^{2}}{2}\right)},
$$

where $\omega_{0}$ is the frequency of wavelet (here $\omega_{0}=5$ ).

In order to compare the spectrum of the initial signals with that of temperature signal $T(t)$ after substitution in the Eq. (3) correlation coefficient of the spectrum (8) both in frequency and time domain was used.

$$
r_{\text {timefreq }}=\frac{\sum_{j} \sum_{i}\left[\left(W_{T}\left(a_{j}, t_{i}+\Delta t\right)-\overline{W_{T}}\left(a_{j}\right)\right) \cdot\left(W_{P P G}\left(a_{j}, t_{i}+\Delta t\right)-\overline{W_{P P G}}\left(a_{j}\right)\right)\right]}{\sqrt{\sum_{j} \sum_{i}\left(W_{T}\left(a_{j}, t_{i}+\Delta t\right)-\overline{W_{T}}\left(a_{j}\right)\right)^{2} \cdot \sum_{j} \sum_{i}\left(W_{P P G}\left(a_{j}, t_{i}+\Delta t\right)-\overline{W_{P P G}}\left(a_{j}\right)\right)^{2}}},
$$

where $W_{P P G}$ and $W_{T}$ are the wavelet spectrums of blood flow determined by photoplethysmography $\omega_{P P G}(t)$ and the temperature $\left(\mathrm{T}(\mathrm{t})\right.$ or $\left.\omega_{\mathrm{T}}(\mathrm{t})\right)$, respectively, $\overline{W_{P P G}}\left(a_{j}\right)$ and $\overline{W_{T}}\left(a_{j}\right)$ are the mean wavelet coefficient values in the scale $\mathrm{a}_{\mathrm{j}}$, $\mathrm{I}$ is the time counts, $\mathrm{j}$ is the scale counts and $\Delta \mathrm{t}$ is the time shift.

By means of Eq. (8) the spectrum of the blood flow signal was compared with the spectrum of the temperature signal $T(t)$ and with the blood flow signal reconstructed from temperature $\omega_{T}(t)$.

\section{Results}

\subsection{Time domain}

Figure 3 shows an example of the signals processed in time domain in accordance with the diagram in Figure 2. Figure $3 a$ shows the initial temperature and PPG signals, Figure 3b shows maxima envelope of pulse waves extracts from the PPG signal, and added interpolation (steps 1-3 in Figure 2), Figure 3c shows the temperature signal processed by its substitution in the Eq. (3), and the signal of the photoplethysmogram (steps 1-6 in Figure 2). Then we compared the blood flow signal determined by temperature and by photoplethysmography (step 7 in Figure 2).

Table 2 shows results of the blood flow restoration for 11 healthy volunteers. Correlation coefficients in table 2 are calculated for different cases: 1.1 initial scaled signals (before processing), 1.2 addition of a time shift to the temperature signal (delay determination), 1.3 temperature signal transformation by expression (3) at $\Delta t=0$ and 1.4 applying expression (3) to the temperature signal with time delay determination. As analyzed signals have complicated frequency contents, determined delay time between the temperature and blood flow signals is a value averaged within a

\begin{tabular}{|c|c|c|c|c|c|c|c|c|c|c|c|c|}
\hline Subject № & 1 & 2 & 3 & 4 & 5 & 6 & 7 & 8 & 9 & 10 & 11 & $\begin{array}{l}\text { Avg. } \\
\text { value } \\
\end{array}$ \\
\hline \multicolumn{13}{|l|}{ 1. Correlation coefficient, $r_{\text {time }}$} \\
\hline 1.1 Before processing & 0.8 & 0.32 & 0.52 & 0.25 & 0.29 & 0.18 & 0.41 & 0.27 & 0.19 & 0.3 & 0.35 & 0.35 \\
\hline 1.2 Delay determination only & 0.82 & 0.42 & 0.61 & 0.42 & 0.34 & 0.34 & 0.61 & 0.53 & 0.26 & 0.42 & 0.54 & 0.48 \\
\hline 1.3 Applying of Eq. (3) only $(\Delta t=0)$ & 0.53 & 0.73 & 0.46 & 0.64 & 0.5 & 0.27 & 0.34 & 0.47 & 0.18 & 0.68 & 0.62 & 0.49 \\
\hline $\begin{array}{c}\text { 1.4 Delay determination and } \\
\text { applying of Eq. (3) }\end{array}$ & 0.53 & 0.73 & 0.58 & 0.65 & 0.52 & 0.7 & 0.68 & 0.84 & 0.27 & 0.72 & 0.71 & 0.63 \\
\hline 2. Average time delay $\Delta t, s$ & 7.5 & 8.5 & 10 & 10.5 & 12 & 15 & 16 & 15 & 16 & 17 & 21 & 13.5 \\
\hline
\end{tabular}
whole signal frequency band.

Table 2. Time domain analysis results for 11 healthy volunteers 

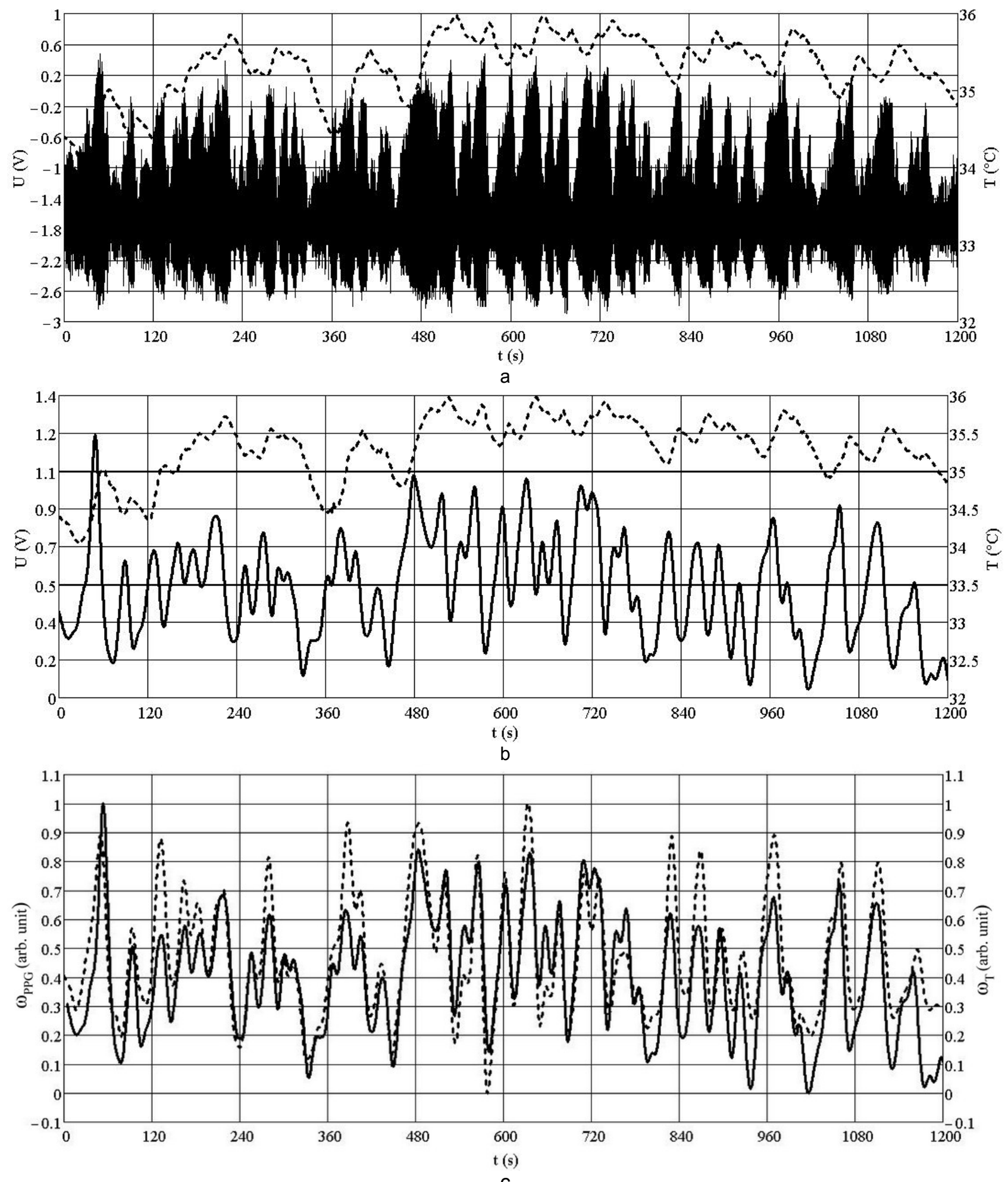

$\mathrm{C}$

Fig. 3. Example of the signal processing of finger temperature variation - $T(t)$ (dotted line) and photoplethysmogram PPG (solid line) for subject №8: $a$ - initial signals $T(t)$ and PPG; $b$ - signal of $T(t)$ and maxima envelope of PPG, $c-$ scaling blood flow signals, calculated from $T(t)$ and $P P G$ (with addition of the time shift $\Delta t$ in the $\omega_{T}(t)$ ) 


\subsection{Time-frequency domain}

Figure 4 shows the time-frequency spectrum of temperature oscillations (Figure 4a), blood flow oscillations estimated by photoplethysmography (Figure 4b) and blood flow reconstructed from the temperature signal (Figure 4c). Spectrums in figure 4 corresponded to the same subject that time series in figure 3.

Table 3 shows the results of determination of correlation coefficients of time - frequency spectrums (expression 8 ) in the neurogenic frequency band $0.02-0.05 \mathrm{~Hz}$, marked with a white rectangle in figure 4 . Correlations in table 3 were calculated taking into account $\Delta \mathrm{t}$-time shift of the temperature data (see table 2).

Table 3. Correlation coefficients of time-frequency spectrums in the neurogenic frequency band 0.02-0.05 Hz: a) correlation between a blood flow estimated by PPG and by the temperature signal, b) correlation between blood flow estimated by PPG and signal reconstructed from the temperature

\begin{tabular}{|l|c|c|c|c|c|c|c|c|c|c|c|c|}
\hline Subject № & $\mathbf{1}$ & $\mathbf{2}$ & $\mathbf{3}$ & $\mathbf{4}$ & $\mathbf{5}$ & $\mathbf{6}$ & $\mathbf{7}$ & $\mathbf{8}$ & $\mathbf{9}$ & $\mathbf{1 0}$ & $\mathbf{1 1}$ & $\begin{array}{c}\text { Avg. } \\
\text { value }\end{array}$ \\
Correlation coef. , $r_{\text {timefreq }}$ & & & & & & & & & & & & \\
\hline a) before transformation (3) & 0.58 & 0.66 & 0.21 & 0.72 & 0.42 & 0.67 & 0.57 & 0.75 & 0.27 & 0.34 & 0.6 & $\mathbf{0 . 5 5}$ \\
\hline b) after transformation (3) & 0.74 & 0.74 & 0.3 & 0.77 & 0.5 & 0.81 & 0.81 & 0.86 & 0.38 & 0.39 & 0.79 & $\mathbf{0 . 6 8}$ \\
\hline
\end{tabular}

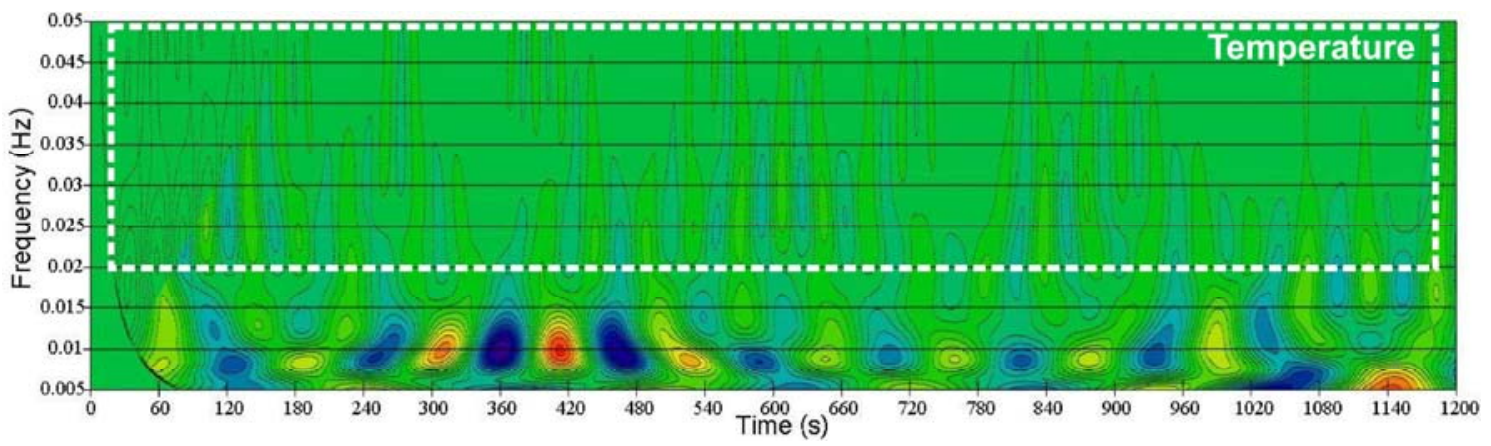

a
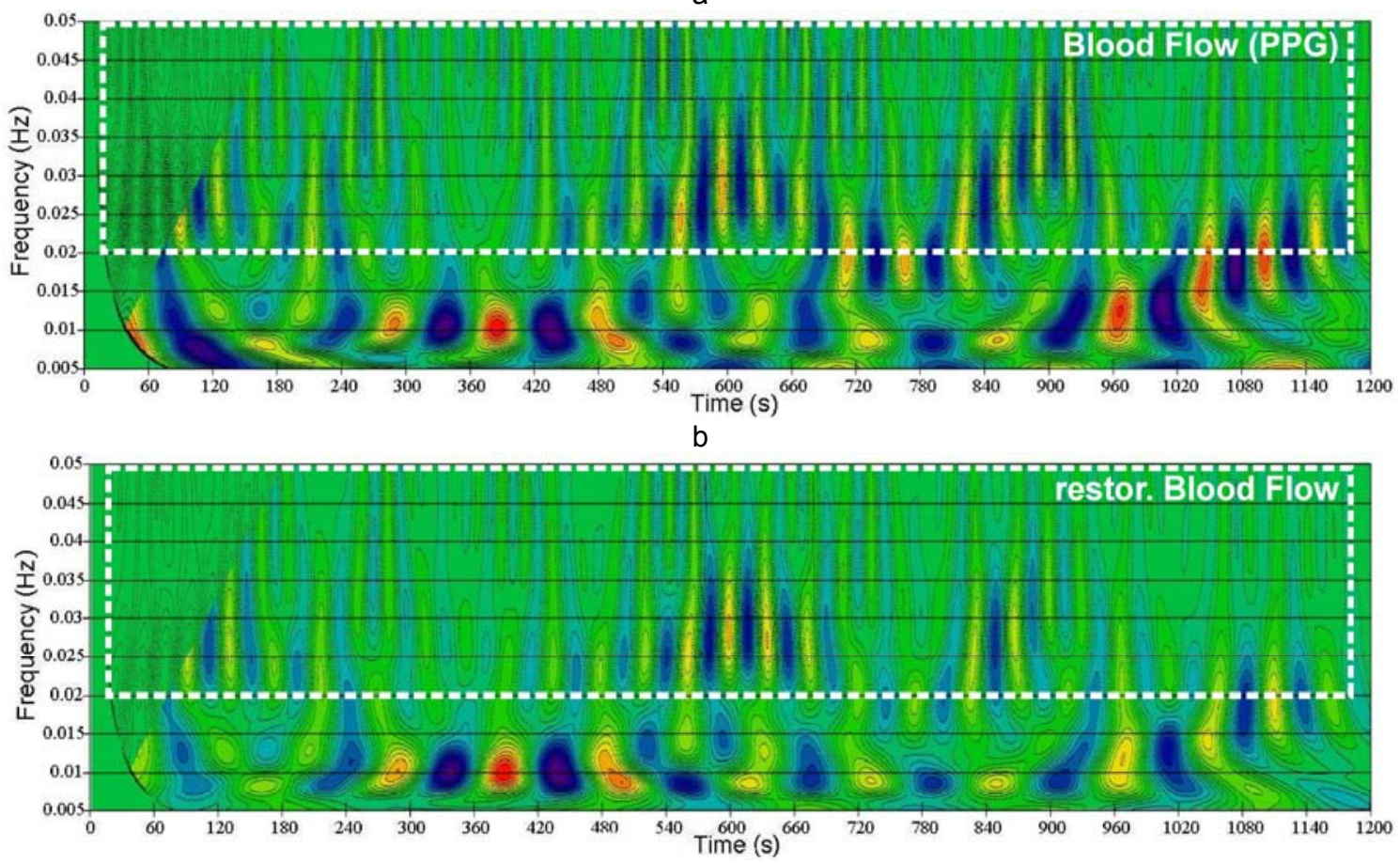

$\mathrm{c}$

Fig. 4. Time-frequency wavelet spectrums: a - finger temperature oscillations, $b$ - blood flow oscillations estimated by $P P G$ and c - blood flow oscillations reconstructed from the temperature signal (subject № 8). White rectangle marked neurogenic range of the blood flow oscillations 


\section{Discussion}

Time series of analysed data in figure 2, c clearly show restoration of the blood flow waveform by substitution of the temperature signal to the expression (3). From table 2 it is evident that the highest correlation of the blood flow and restored blood flow corresponds to the case when we use formula (3) with delay determination (table 2, case 1.4). An average value of the correlation coefficient increases from 0.35 for the initial signals to 0.63 for the processed signals (cases 1.1 and 1.4 in table 2). It proves good possibilities of restoration of a finger blood flow oscillations by means of temperature data and the fact that finger temperature oscillations depend to a great extent upon blood flow oscillations (at least at rest conditions). Specified value of the delay time caused by finite time of skin heating and cooling during transferring of thermal energy from blood to skin surface which has some heat capacity.

Results of time- frequency wavelet analysis of the signals show similarity of blood flow and temperature oscillation components in endothelial frequency interval below $0.02 \mathrm{~Hz}$ (figure $4 \mathrm{a}$ and b). Reconstruction of blood flow oscillations by means of temperature data and expression (3) leads to restoration of spectral components of neurogenic frequency band $0.02-0.05 \mathrm{~Hz}$ (see rectangle area in figure $4 \mathrm{c}$ ). Comparison of wavelet spectrum correlations in table 3 also reveals that after applying expression (3) we can observe restoration of blood flow oscillations in neurogenic frequency band.

Therefore, it is likely that blood flow oscillations in endothelial frequency band can be investigated by observing temperature oscillations without transformation of temperature data with the exception of scaling and delay determination. For investigations of higher neurogenic frequency components of blood flow it is necessary to transform temperature oscillations, for example, by means of expression (3).

Considered method of blood flow restoration by means of temperature measurements can be useful in phase coherence investigations [7-10] and for development of non-contact methods of blood flow observations. The latter is the most important taking into consideration that a temperature response to trials (cooling of extremities, for example) can be more evident than blood flow response [7].

Most likely, finger temperature oscillations and blood flow oscillations should be considered as closely related values; temperature variations regulation (at least at rest conditions) is almost completely subordinated to blood flow regulation. Herewith, it is necessary to take into account sweet glands activity and skin thermal properties variations.

\section{Conclusion}

Considered method of restoration of blood flow oscillations from temperature oscillations measured by thermal imaging camera shows a good possibility of waveform reconstruction (figure 3c and table 2 ), as well as reconstruction of time-frequency spectrum of blood flow oscillations especially in neurogenic frequency band $0.02-0.05 \mathrm{~Hz}$ (figure 4c and table 3).

Oscillations of blood flow and temperature of finger tips have a similar form in time - frequency domain of endothelial activity (below $0.02 \mathrm{~Hz}$ ). It is most likely to study endothelial blood flow oscillations by means of analyzing finger tips temperature oscillations and perhaps methodically it is easier than direct blood flow analysis. Using temperature data in order to study blood flow oscillations in neurogenic activity frequency band required additional processing, for example, via viewed method of blood flow restoration by expression (3).

Findings of this investigation can serve as the proof of presence of time shift between temperature and blood flow oscillations. Temperature data lags from blood flow values and has a time delay specific for each subject (delay values varies approximately from 7 to 20 seconds). Thus, blood flow oscillations of finger tips at rest can be restored by means of amplitude transformation of temperature signal (3), and time transformation (shift for the delay time).

The considered method of the non-contact determination of blood flow oscillations by the temperature measurements can find practical application in investigations of thermal skin damage, investigation of physical and chemical impacts on the circulatory dynamics in the peripheral vessels and skin microcirculation.

\section{REFERENCES}

[1] Burton A. C., Taylor R. M., "A study of the adjustment of peripheral vascular tone to the requirement of the regulation of body temperature". Am. J. Physiol., vol. 129, pp. 566-577, 1940.

[2] Shusterman V., Anderson K. P., Barnea O. "Spontaneous temperature oscillations in normal human subjects". Am. J. Regul. Integr. Comp. Physiol. vol. 273, pp. 1173 - 1181, 1997.

[3] Trafford J., Lafferty K. "What does photoplethysmography measure?". Medical and biological engineering and computing, vol. 22, pp. 479-480, 1984.

[4] Chan G., S. H., Middleton P. M., Lovell N. H., Celler B. G., "Extraction of photoplethysmographic waveform variability by lowpass filtering". Conf. Proc. IEEE Eng. Med. Biol. Soc. vol. 5, pp. 5568-5571, 2005.

[5] Ley O., Deshpande C.V. "Comparison of two mathematical models for the study of vascular reactivity". Computers in Biology and Medicine. vol.39, №7, pp. 579-589, 2009.

[6] Bracic M., Stefanovska A. "Wavelet-based analysis of human blood-flow dynamics”. Bull Math Biol. vol. 60, №5, pp. 919-935, 1998.

[7] Bandrivskyy A., Bernjak A., McClintock P., at al. "Wavelet phase coherence analysis: application to skin temperature and blood flow". Cardiovascular engineering: an international journal. vol. 4, №1, pp. 89-93, 2004. 
[8] Podtaev S., Morozov M., Frick P. "Wavelet - based correlation of skin temperature and blood flow oscillations" Cardiovasc. Eng. vol. 8, pp. 185-189, 2008.

[9] Sheppard L.W., Vuksanovic V., Stefanovska A., et al. "Oscillatory dynamics of vasoconstriction and vasodilation identified by time-localized phase coherence". Physics in medicine and biology. vol. 56, pp. 3583-3601, 2011.

[10] Liu W., Meyer J., Scully C., at al. "Observing temperature fluctuations in humans using infrared imaging". QIRT Journal. vol. 8, №1, pp.21-36, 2011. 